\title{
Enhancing Physical Resolution of Constructed Ladder Network for an Interleaved Winding
}

\author{
Saurav Pramanik \\ HV Lab, Dept. of Electrical Engineering \\ Indian Institute of Science, Bangalore \\ India \\ saurav.pramanik@gmail.com
}

\author{
L. Satish \\ HV Lab, Dept. of Electrical Engineering \\ Indian Institute of Science, Bangalore \\ India \\ satish@hve.iisc.ernet.in
}

\begin{abstract}
The number of detectable natural frequencies in a frequency response governs the number of sections of the ladder network that can be constructed, starting from the measured frequency response data. This number, particularly in case of interleaved windings, is woefully small rendering the entire exercise pointless. An attempt is described wherein this physical resolution can be enhanced. This is achieved by augmenting the measured driving-point admittance data (of the interleaved winding) with information regarding natural frequencies that are observable, and hence extractable, from an additional measurement of the open-circuited neutral-end voltage spectrum. By this process, a few more additional natural frequencies become available for processing, resulting in a ladder network with an increased physical resolution, i.e., more number of sections. Measurements done on an actual interleaved winding are presented to demonstrate feasibility of the proposed method.
\end{abstract}

Keywords-Interleaved winding, natural frequency, ladder network, frequency response, rational function

\section{INTRODUCTION AND MOTIVATION}

There exist methods [1, 2, 3] to construct physically realizable, inductively coupled, ladder networks to model and/or represent a single isolated uniformly wound transformer winding, starting from its measured frequency response. The number of sections in the synthesized ladder network is directly dependant on the number of natural frequencies observable in the frequency response and, this inturn is a direct measure of the physical resolution afforded by the constructed ladder network. Higher the physical resolution achieved, greater will be the accuracy of mapping the physical winding to the synthesized circuit. One possible application would be for localization of mechanical damages in the winding. Sometimes, this resolution is severely limited because, not all natural frequencies are always excitable in a given measurement configuration and terminal connection.

Typically, it is desirable to construct a ladder network wherein at least one or two pair of disks would correspond or map onto one section of the ladder network. Although, in case of the continuous-disk windings this resolution achieved is higher (as relatively a larger number of natural frequencies are observable in its frequency response plot), the same is certainly not the case for interleaved-disk windings, wherein a maximum of 2-3 natural frequencies are observable $[4,5]$. So, the physical resolution achievable, especially for interleaved windings, by this method of approach is very poor, thus, defeating the very purpose of this exercise. So, any work by which this resolution can be improved is naturally worthwhile to pursue. This was the motivation.

\section{BASIC PHILOSOPHY}

\section{A. Principle}

In an earlier work [5], it was found that the normally suppressed natural frequencies in an interleaved-disk winding when seen through its line or neutral current, were rendered excitable when seen in its disk-to-disk voltages. This was a unique feature of the interleaved winding's behaviour. But, for obvious reasons, it is normally not possible to access the diskto-disk voltages. Nevertheless, taking this feature as a clue, it was decided to explore if the same trend in behaviour might also be observable, if the voltage of the last disk, namely, the neutral-end was considered. To this effect, a ratio of opencircuited neutral-end voltage spectrum to input voltage spectrum was considered. Henceforth, this ratio will be referred to as the voltage ratio spectrum. Intuitively, it seemed likely, but, actual measurements were made to verify this fact. Just as in disk-to-disk voltages, here also, measurements revealed presence of an increased number of natural frequencies. The basic philosophy of this paper is to gather this additional information and suitably use it to achieve an improved physical resolution of the constructed ladder network.

\section{B. Procedure}

The following steps briefly outline the procedure to implement the proposed method:

- $\quad$ Measure DPA (Driving Point Admittance) frequency response.

- Measure the voltage ratio spectrum (with neutral open). From this plot, carefully extract (after zooming) all frequencies corresponding to the peaks in it. Append these natural frequencies (i.e. poles) to the DPA response beyond the last observed DPA peak. Call the appended DPA as ADPA.

- Using DPA data and the genetic algorithm (GA) method (see [3] for details), determine a ladder network corresponding to the DPA. 
- $\quad$ Thereafter, using ADPA and GA method, determine a new ladder network that accurately reproduces both the measured DPA and ADPA (poles and zero).

- Output the resulting ladder network, which has more number of sections than the visible peaks in the DPA frequency response

Thus, with the additional neutral-end voltage measurement it has been possible to construct a ladder network that has enhanced physical resolution.

(Note: Information about 'zeros' of ADPA corresponding to the appended 'poles' is unavailable from the voltage ratio spectrum. Given the fact that these natural frequencies are rendered non-excitable in the DPA function, it is appropriate to initially assign a value to 'zero' which is numerically very close to the immediate neighboring 'pole' [5]. Furthermore, while doing so, the alternating arrangement of pole and zero must be strictly adhered to. With these precautions taken, the GA method is able to accurately predict the value of zero so as to satisfy the objective function.)

\section{EXPERIMENTAL RESULTS}

The above procedure was implemented on an actual single isolated interleaved-disk winding. The winding was air-cored and had total 16 disks with 10 turns per disk, height of 225 $\mathrm{mm}$, inner and outer diameter of $260 \mathrm{~mm}$ and $350 \mathrm{~mm}$ respectively. Each copper turn had a cross-section of $10 \mathrm{~mm} \mathrm{x}$ $1.8 \mathrm{~mm}$. An Al-foil was concentrically placed on the inner surface of the winding to form the reference ground plane. All the responses were measured using sweep frequency method employing (i) an arbitrary waveform generator, capable of producing a maximum $20 \mathrm{~V}_{p} p$ sinusoid with frequency range of 0-80 MHz, (ii) a $200 \mathrm{MSa} / \mathrm{s}, 8$-bit digital oscilloscope and (iii) a clamp on current probe with sensitivity of $2 \mathrm{~mA} / \mathrm{mV}$, bandwidth: $450 \mathrm{~Hz}-60 \mathrm{MHz}$ and (iv) a 50 coaxial cable. Experimental setup is shown in Fig. 1.

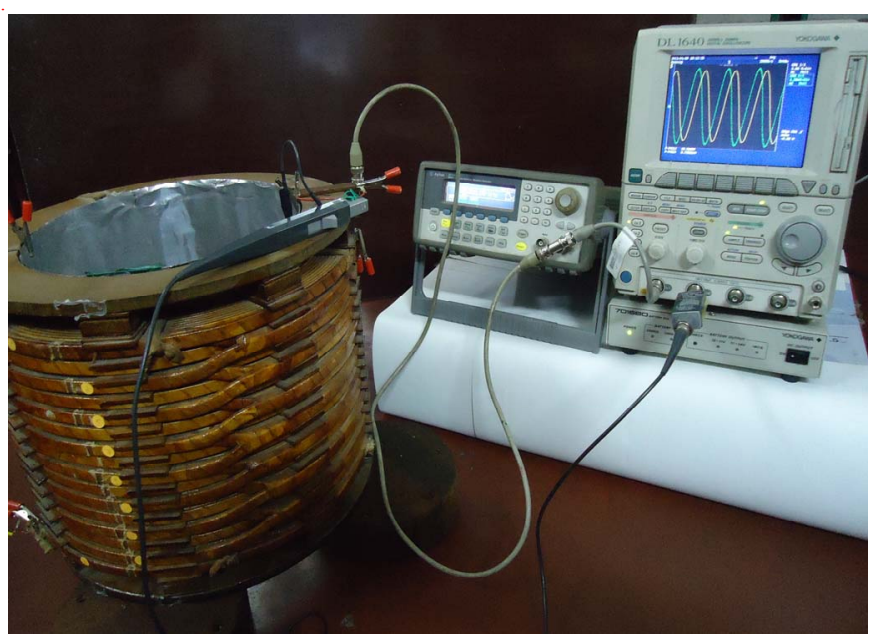

Fig. 1. Experimental setup

Measured responses are-

1. DPA magnitude response (Fig. 2) and
2. Magnitude of voltage ratio spectrum (Fig. 3)

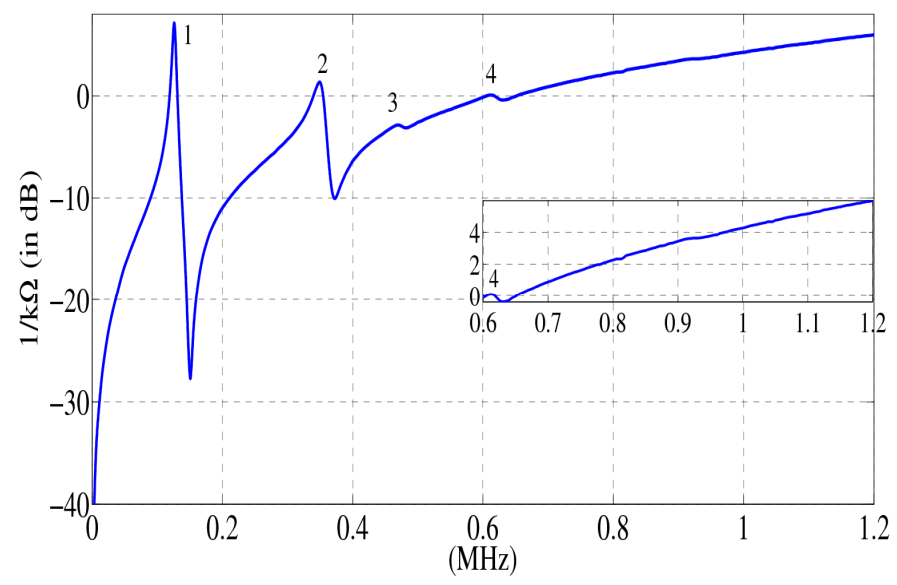

Fig. 2. Measured DPA magnitude of interleaved-disk winding

Salient features observable in Figs. 2 and 3 are listed below-

1. Fig. 2 reveals 4 peaks, while 7 are observable in Fig.3.

2. All resonances (peaks) for both the line-current and neutral-end voltage occur at the same frequencies viz. the natural frequencies of the system.

3. Initial four resonance peaks are distinctly observable in both DPA (i.e. line-current) and voltage ratio spectrum, while, the last three peaks that are detectable in voltage ratio spectrum (in zoomed plot) are conspicuously missing in DPA even after zooming.

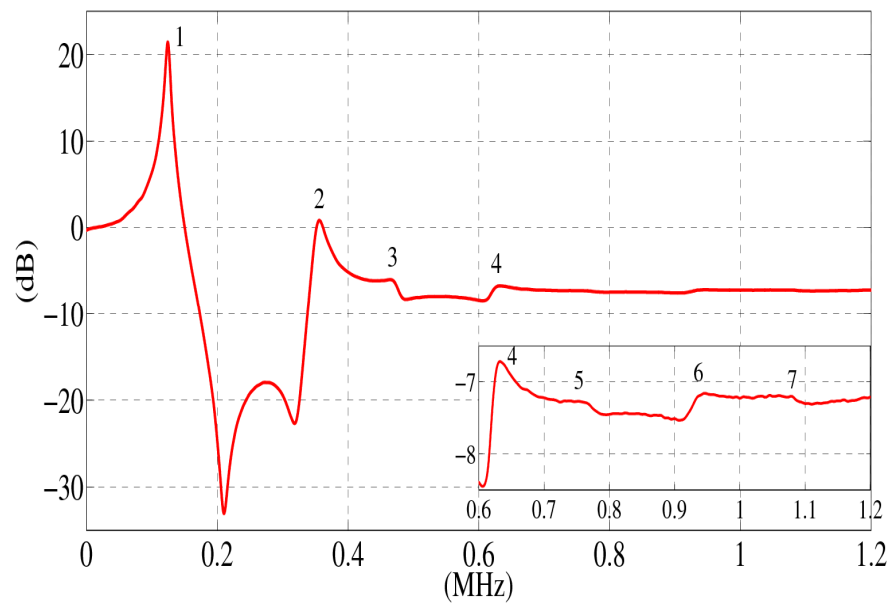

Fig. 3. Measured voltage ratio spectrum of an interleaved-disk winding

Thus, the sole use of measured voltage ratio spectrum is to extract these hidden natural frequencies (i.e. the pole frequencies) that were non-excitable in the line current response (i.e. the DPA magnitude), and construct an equivalent circuit with more number of sections. Here, a 7section model was constructed compared to a 4-section model. Unlike the DPA, the high frequency poles in the voltage ratio spectrum are not closely associated with their neighboring zeros which would cancel the corresponding resonance peaks [5] and hence, three additional peaks are observable in Fig. 3. The task of constructing the ADPA, which is the first step of 
constructing the model, was performed following the steps below-

1. Initially the DPA magnitude response was considered and GA was used to fit the response to a rational function of four complex conjugate pair of poles and zeros.

2. Later $5^{\text {th }}, 6^{\text {th }}$ and $7^{\text {th }}$ pole frequencies were identified from the measured voltage ratio magnitude spectrum (zoomed version) in Fig. 3 and the associated zeros i.e. the trough frequencies were estimated using GA. The following unique properties of DPA were used as constraints in implementing the GA method-

$>$ Every pole is associated with a zero and this zero frequency is always greater than the corresponding pole frequency (i.e. $\mathrm{z}_{\omega}>\mathrm{p}_{\omega}$ ).

$>$ For a uniformly wound winding, pole-zero separation distance gradually reduces with the frequency [4] and therefore, accordingly the lower and upper limit of $z_{\omega}$ was fixed. It is evident from Fig. 2 that DPA peaks at the pole frequencies gradually decay with the frequency. At higher frequencies, the zeros are positioned closer to the corresponding poles and this along with the damping effect, explains why DPA peak magnitude decays gradually. Network analysis and the experimental results in [4] elaborately explains this fact.

$>$ Damping effect is more for high frequency poles and zeros, and hence, the real parts of any polezero pair is always greater than its preceding pair. This property was used for limiting the search space for real parts of poles and zeros.

Finally, the appended DPA was constructed to match the magnitude response. Results are shown in Table I in polezero-gain format.

TABLE I.

ADPA IN P-Z-K FORMAT

\begin{tabular}{|c|c|c|c|}
\hline$\#$ & Poles (P) & Zeros $(\mathrm{Z})$ & Gain (K) \\
\hline & & 0 & \\
\cline { 1 - 3 } 1 & $-0.0183 \pm 0.7935 \mathrm{i}$ & $-0.0201 \pm 0.9454 \mathrm{i}$ & \\
\cline { 1 - 3 } 2 & $-0.0607 \pm 2.2205 \mathrm{i}$ & $-0.0618 \pm 2.3118 \mathrm{i}$ & \\
\cline { 1 - 3 } 3 & $-0.0838 \pm 2.9835 \mathrm{i}$ & $-0.0839 \pm 2.9909 \mathrm{i}$ & \multirow{2}{*}{$\mathbf{3 . 6 8 3 8}$} \\
\cline { 1 - 3 } 4 & $-0.0896 \pm 3.9118 \mathrm{i}$ & $-0.0882 \pm 3.9207 \mathrm{i}$ & \\
\cline { 1 - 3 } 5 & $-0.1202 \pm 4.8395 \mathrm{i}$ & $-0.1185 \pm 4.8400 \mathrm{i}$ & \\
\cline { 1 - 3 } 6 & $-0.1352 \pm 5.8780 \mathrm{i}$ & $-0.1336 \pm 5.8800 \mathrm{i}$ & \\
\cline { 1 - 3 } 7 & $-0.1464 \pm 6.8260 \mathrm{i}$ & $-0.1430 \pm 6.8280 \mathrm{i}$ & \\
\hline
\end{tabular}

Table I clearly shows that all the constraints described above for pole-zero estimation are completely satisfied. Fig. 4 shows an excellent match between the measured and fitted responses for DPA and ADPA simultaneously.
Once the pole-zero frequencies are available from the constructed DPA/ADPA, following the method in $[3,6]$, the capacitances, inductances and resistances were determined. First, a 4-section model was constructed from the estimated DPA followed by a 7-section model from the ADPA. For brevity, only a summary of the results are given below.

1. Shunt Capacitance Measurement $\left(C_{g}\right)$ : Measured DPA $(|\mathrm{Y}|)$ at $10 \mathrm{kHz}$ with the neutral-end open gives a total $C_{g}$ of $0.457 \mathrm{nF}\left(C_{g}=|\mathrm{Y}| /(2 \pi \mathrm{f})\right)$.

2. Series Capacitance $\left(C_{s}\right)$ Estimation: $C_{s}$ estimation was done for both 4-section and 7-section models and is shown in Table II. Method described in [6] was followed to estimate $C_{s}$. Here, the fitted ADPA for 7section model also estimated a $C_{s}$ value that is very close to that estimated for the 4-section model.

(a)

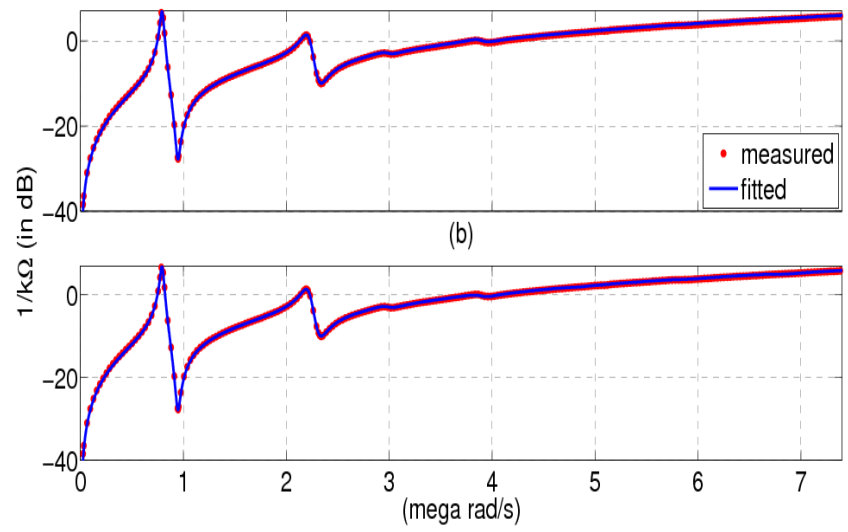

Fig. 4. Measured and fitted magnitude response (a) DPA for 4-section model with ' $\mathrm{K}$ '=3.6629 (b) ADPA for 7-section model with ' $\mathrm{K}$ '=3.6838.

TABLE II. $\quad C_{S}$ ESTIMATION

\begin{tabular}{|l|l|}
\hline 4-section model (DPA) & 7-section model (ADPA) \\
\hline${ }^{\prime} \mathrm{K}$ ' $=3.6629$ & ' $\mathrm{K}$ ' $=3.6838$ \\
\hline Total $C_{s}=0.188$ & Total $C_{s}=0.191$ \\
\hline
\end{tabular}

3. Estimation of Inductances using GA: With the above $C_{s}$ and $C_{g}$ values known, first a lossless model was constructed using GA. Objective or error function (' $g$ ') used for this purpose is (i.e. matching the pole-zero frequencies) given below

$$
g=\frac{\sum_{i=1}^{n}\left(\omega_{p m i}-\omega_{p e i}\right)^{2}+\sum_{i=1}^{n}\left(\omega_{z m i}-\omega_{z e i}\right)^{2}}{2 n}
$$

where, subscripts $\mathrm{p}$ and $\mathrm{z}$ refer to the pole and zero, while $\mathrm{m}$ and e refer to the measured and estimated terms respectively. ' $n$ ' refers to the number of resonant peaks or natural frequencies. The inequality constraints for self and mutual inductances used in GA are given below.

$$
\mathrm{L}_{11}>\mathrm{L}_{12}>\mathrm{L}_{13}>\ldots .>\mathrm{L}_{1 \mathrm{n}}
$$


2013 IEEE 1st International Conference on Condition Assessment Techniques in Electrical Systems

$$
\begin{aligned}
& \left(\mathrm{L}_{11}-\mathrm{L}_{12}\right)>\left(\mathrm{L}_{12}-\mathrm{L}_{13}\right)>\ldots>\left(\mathrm{L}_{1(\mathrm{n}-1)}-\mathrm{L}_{1 \mathrm{n}}\right) \\
& \mathrm{L}_{\mathrm{ij}}=\mathrm{L}_{\mathrm{ji}}, \mathrm{L}_{\mathrm{ii}}=\mathrm{L}_{\mathrm{jj}} \text { and } \mathrm{L}_{\mathrm{i}, \mathrm{j}}=\mathrm{L}_{\mathrm{i}+1, \mathrm{j}+1}
\end{aligned}
$$

where $L_{i j}=$ mutual inductance between $i^{\text {th }}$ and $j^{\text {th }}$ section.

These inequalities assist in enforcing construction of a physically realizable ladder-network. Further, it also reduces the search space. Considering all the sections to be identical, the algorithm is able to meet the above inequalities for each and every update of the variables. Details of GA application for this task are elaborated in [3]. Only the important aspects are included here. Table III shows the inductances for 4-section and 7section model respectively.

TABLE III. ESTIMATED INDUCTANCES

\begin{tabular}{|c|c|c|}
\hline $\begin{array}{c}\text { Inductances } \\
(\mathrm{mH})\end{array}$ & $\begin{array}{c}\text { 4-Section } \\
\text { model }\end{array}$ & $\begin{array}{c}\text { 7-Section } \\
\text { model }\end{array}$ \\
\hline$L_{11}$ & 0.4067 & 0.1424 \\
\hline$L_{12}$ & 0.2892 & 0.1115 \\
\hline$L_{13}$ & 0.2272 & 0.0882 \\
\hline$L_{14}$ & 0.2119 & 0.0782 \\
\hline$L_{15}$ & - & 0.0725 \\
\hline$L_{16}$ & - & 0.0694 \\
\hline$L_{17}$ & - & 0.0673 \\
\hline $\boldsymbol{L}_{\text {eq }}$ & $\mathbf{4 . 6 9 4 7}$ & $\mathbf{4 . 6 9 0 7}$ \\
\hline
\end{tabular}

Although no constraint was used for $L_{e q}$ in GA, but still the estimation converged to the same $L_{e q}$ for both the cases. The present estimation is proved to be accurate since the change in number of sections did not alter the total effective winding inductance. Computed responses from the estimated models are plotted below along with the measured response in Fig. 5, which is further proof of the accuracy achieved by the proposed method.

4. Evaluation of Resistance: In the last step, the resistance of the winding was estimated to match the magnitude of the measured DPA. Hence, the error function was suitably modified to be the difference between the measured and estimated magnitude and redefined as-

$$
g=\frac{\sqrt{\sum_{i=1}^{p}\left(M_{m i}-M_{e i}\right)^{2}}}{p}
$$

where $p=$ length of DPA magnitude data $M$.

Total resistance for the winding was found $175.88 \Omega$ and $175.7 \Omega$ respectively for 4 and 7 -section model. Computed models are shown in Fig. 5. Extent of match in magnitude with and without the resistance included is clearly seen from Fig. 6.
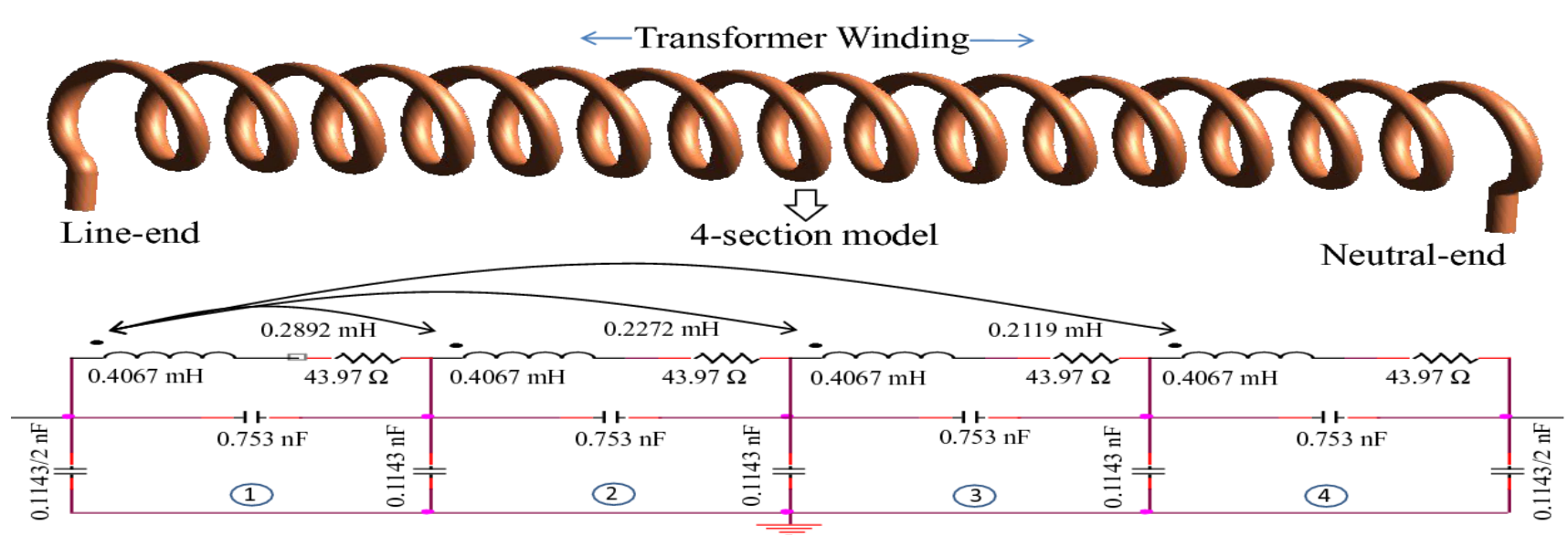

7-section model

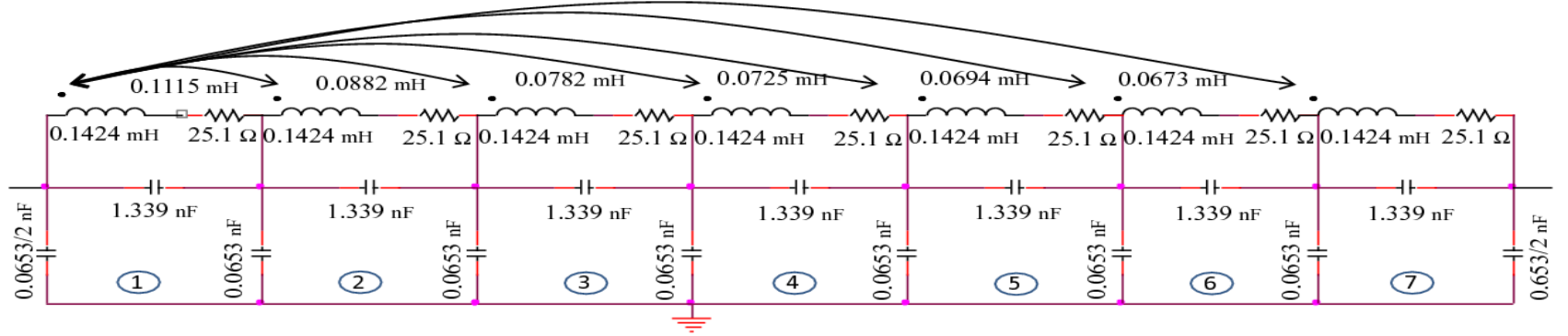

Fig. 5. Four and seven section model of the actual single isolated interleaved winding. 
2013 IEEE 1st International Conference on Condition Assessment Techniques in Electrical Systems

(a)

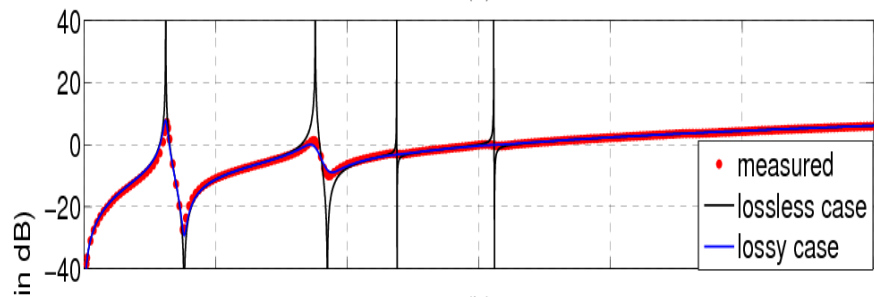

(b)

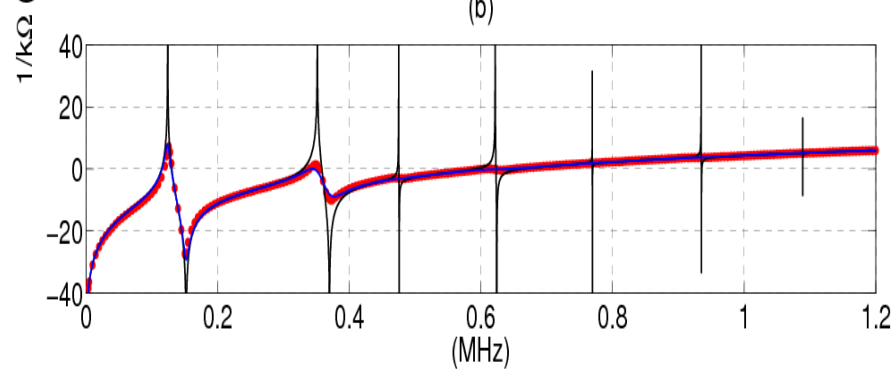

Fig. 6. Measured and computed DPA for (a) 4-section model and (b) 7section model (with and without losses).

Further, the estimated model was also validated by measuring the other terminal responses viz. the transfer admittance (TA) and the terminal voltage ratio spectrum. Initially, neutral was grounded and TA (transfer admittance) was computed for both 4-section and 7-section model. Later on, neutral-end voltage was computed with the neutral-end kept open. All these computed responses are plotted along with the corresponding measured responses in Figs. 7 and 8. The extent of match is evident and can be considered to be within acceptable limits. The extent of match obtained shows that physical realizability of the winding achieved by the 4section model is not only preserved in the 7-section model, but, in addition its physical resolution has also been enhanced (see Fig. 5).

(a)

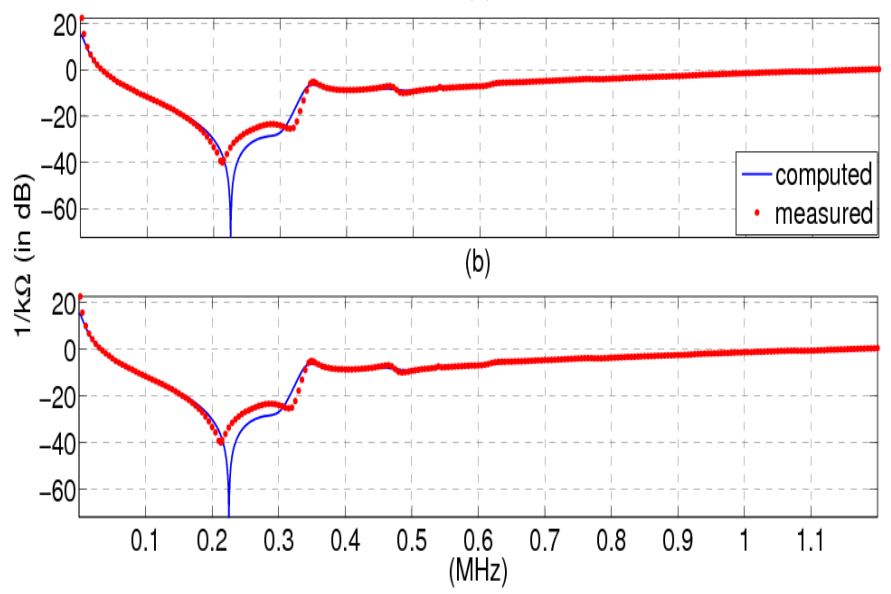

Fig. 7. Measured and computed TA with neutral grounded (a) 4-section model (b) 7-section model

Pertinent nodes of the winding corresponding to the 7-section model were not physically accessible from the outside and hence node voltages could not be measured to compare with the computed values. However, for the 4-section model all nodes were physically accessible from outside since each section mapped to every four disks of total 16 disks. Here, node voltages were measured and could be compared with the computed values. The comparison is shown in Fig. 9. Extent of match proves the validity of the adopted method as well as the suitability of the constructed model for internal inspection of the winding. Hence, the 7-section model constructed by following the same method would definitely represent the internal responses of the winding, while offering a higher physical resolution.

(a)

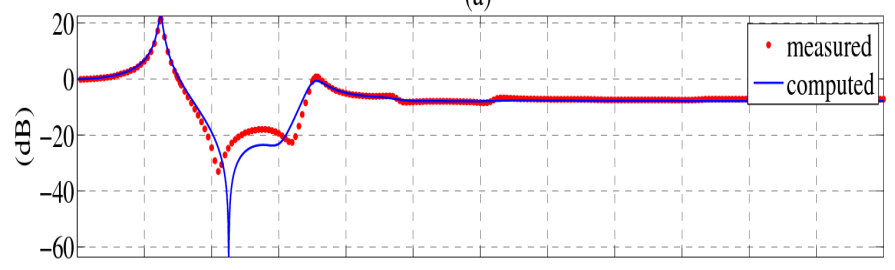

(b)

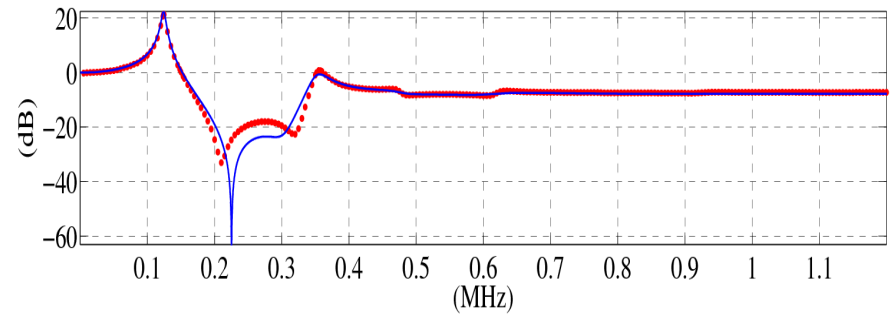

Fig. 8. Measured and computed spectrum of the voltage ratio (neutral end voltage to the input voltage) for (a) 4-section model (b) 7-section model.

(a)

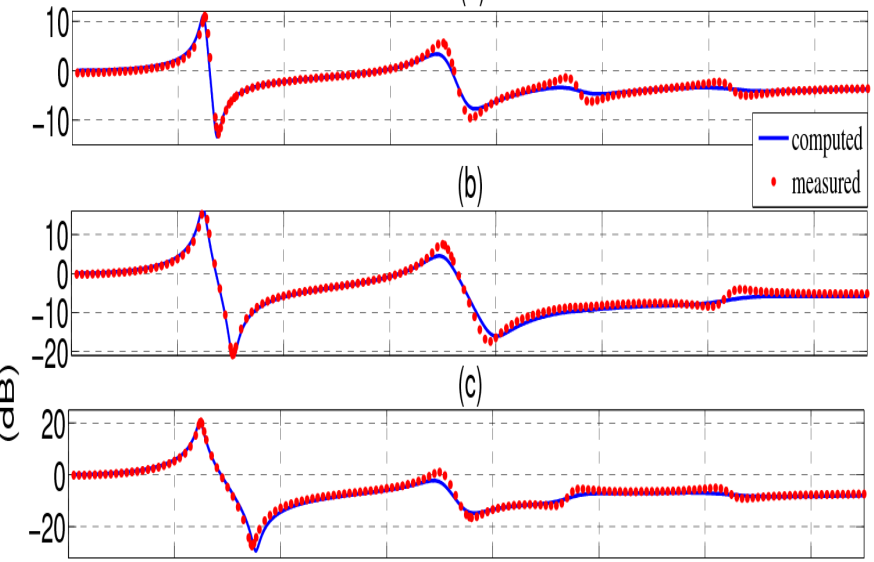

(d)

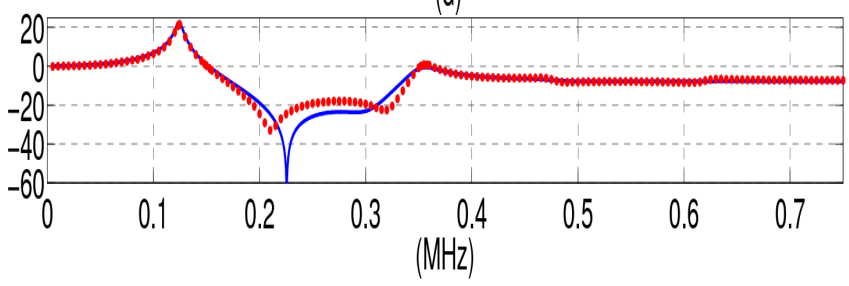

Fig. 9. Measured and computed magnitude spectrum for ratio of node voltage to the input voltage for the 4-section model; (a) $V_{2}(s) / V_{\text {in }}(s)$, (b) $V_{3}(s) / V_{\text {in }}(s)$, (c) $V_{4}(s) / V_{\text {in }}(s)$, (d) $V_{n}(s) / V_{\text {in }}(s)$.

The pole-zero frequencies of the fitted ADPA were used to construct the model and hence, the model also reproduced all the pole-zeros (i.e. the peaks and troughs) in the computed 


\section{IEEE 1st International Conference on Condition Assessment Techniques in Electrical Systems}

DPA (see Fig. 6). It also reproduced the measured peaks for other responses (in Figs. 7, 8 and 9) since all the peaks always occur at the same set of ADPI poles viz. the natural frequencies of the system. The set of zeros for TA and other system functions (ratio of neutral-end or internal node voltages to input voltage) are entirely different from the DPA/ADPA zeros but still the constructed model could estimate them very closely to measured troughs as seen in Figs. 7, 8 and 9 except for minor deviations.

\section{REMARKS}

1. Once the ADPA is constructed in pole-zero-gain format, the estimation process for both inductances and resistances took less than a minute even for 7section model. This proves the method is very fast and time-efficient.

2. The magnitude of the error function chosen to be less than $10 \mathrm{e}-5$ and with this limit was found to always yield a unique set of inductances and resistances. This error limit, as well as, the uniqueness of the model was ensured by increasing the GA population and generation number.

3. Inclusion of frequency dependency of inductances and resistances in future will definitely improve the accuracy provided by the model.

\section{CONCLUSIONS}

This paper describes a novel method, perhaps for the first time, about how the physical resolution of the constructed ladder network can be improved, in particular, for an interleaved winding. This is rendered possible by making an additional frequency response measurement (ratio of the spectra of open-circuited neutral-end voltage to spectra of input) in which a few more natural frequencies are observable, in comparison to the routinely measured driving point impedance/admittance measurement. These few extra frequencies are NOT excitable in the driving point admittance measurement, and hence, in practice only partial information about the winding natural frequencies is available. Experimental results on an actual, single, isolated, uniformly wound, fully interleaved-disk transformer winding are presented. In the example, it is demonstrated how a 4-section ladder network (constructed based on four observable frequencies in driving point admittance data) could be extended to build a 7-section network. This increase is strictly governed by the additional natural frequencies (or peaks) that can be detected in the additional measurement. The extent of agreement between the estimated and measured terminal responses from the 4-section and 7-section networks corresponding to a single isolated interleaved winding clearly demonstrates the feasibility of the method. The new ladder network is not only physically realizable but also offers a higher physical resolution.

\section{REFERENCES}

[1] V. Rashtchi, E. Rahimpour, H. Fotoohabadi, "Parameter Identification of Transformer Detailed Model Based on Chaos Optimisation Algorithm", IET Electr. Power Appl, Vol. 5, Iss. 2, pp. 238-246, 2011.

[2] Pritam Mukherjee and L. Satish, "Construction of Equivalent Circuit of a Single and Isolated Transformer Winding From FRA Data Using the ABC Algorithm", IEEE Trans. Power Del., Vol. 27, No. 2, pp. 963-970, April, 2012.

[3] Saurav Pramanik and L. Satish, "Physical Representation of a Transformer Winding by a Coupled Ladder Network Constructed From its Measured Frequency Response", accepted for presentation in $18^{\text {th }}$ ISH 2013, Seoul, Korea Aug. 2013, pp. 1-6.

[4] Satish, L., Jain, A.: 'Structure of transfer function of transformers with special reference to interleaved windings', IEEE Trans. Power Deliv., 2002, 17, pp. 754-760.

[5] Saurav Pramanik, Sana Anees and L. Satish, "Interleaved Winding and Suppression of Natural Frequencies", IET Electr. Power Appl, Vol. 7 , Issue 4, pp. 237-244, April 2013.

[6] Saurav Pramanik and L. Satish, "Estimation of Series Capacitance of a Transformer Winding Based on Frequency Response Data: An Indirect Measurement Approach", IEEE Trans. Power Del., Vol. 26, No. 4, pp. 2870- 2878, Oct. 2011. 